\title{
Modular forms of weight $1 / 2$ over class number 1 imaginary quadratic number fields
}

\author{
by \\ DAvid Gove (Bakersfield, Calif.)
}

1. Introduction, notation, and statement of results. This paper generalizes a 1977 result of J.-P. Serre and H. M. Stark [3] in which a set of theta functions was proved to be a basis for the space of modular forms of weight $1 / 2$ over $\mathbb{Q}$, which will be referred to as the rational case. Their result has been generalized to forms over totally real fields, and, using representation theory, to arbitrary fields ([1]). Here we show that the original constructive proof can be generalized at least to weight $1 / 2$ forms over the nine imaginary quadratic class number 1 fields. This method will generalize to arbitrary number fields contingent upon the working out of the $p$-adic theta multiplier.

Many of the necessary lemmas and theorems are the same as in the original paper. They are all stated again here, although a corresponding proof will not be included unless it is very short or has a part that is different from [3]. The main difference is that in [3] many of the needed results on the coefficients of modular forms are proved by considering the Dirichlet series associated with the form in question. This trick does not work with modular forms over a number field since more than one coefficient of a form corresponds to each term of the associated Dirichlet series. The method of working directly with the Fourier series used herein would, of course, also work in the rational case. The operators used here are similar to those in [3] but normalized in order to preserve the quaternionic "upper half plane." An added bonus is that this gives them all determinant 1.

Following Stark [5], modular forms of half-integral weight over an arbitrary number field $K$ can be defined in terms of a variable $z$, which comes from a product of upper half planes. In our case, we have

$$
z \in \mathbb{H}=\left\{x+k y \mid x \in \mathbb{C}, y \in \mathbb{R}^{+}\right\},
$$

the quaternionic "upper half plane". This space is preserved by $S L_{2}(\mathbb{C})$, 
with the following action. If $A=\left(\begin{array}{ll}a & b \\ c & d\end{array}\right) \in S L_{2}(\mathbb{C})$ then

$$
A \circ z=(a z+b)(c z+d)^{-1} \text {. }
$$

The order of the action must be specified because of the non-abelian nature of quaternionic multiplication.

Let $O_{K}$ be the integers of the field $K$ and $\chi$ a numerical character (defined on numbers rather than ideals) modulo $N$, which is divisible by 4 . Then the associated theta function is

$$
\theta_{\chi}(z)=\sum_{b \in O_{K}} \chi(b) q\left(b^{2}\right),
$$

where

$$
q(b)=q(b, z)=\exp \left\{2 \pi i \operatorname{tr}\left(t_{0} x b+k y\left|t_{0} b\right|\right)\right\},
$$

with

$$
\operatorname{tr}(x+k y)=x+\bar{x}+2 i y
$$

and $t_{0}$ is a fixed generator of the inverse different $\mathcal{D}^{-1}$ of $K$.

If $\Gamma_{0}(N)=\left\{A=\left(\begin{array}{ll}a & b \\ c & d\end{array}\right) \in S L_{2}\left(O_{K}\right) \mid c \equiv 0(\bmod N)\right\}$, then for any $A \in \Gamma_{0}(N)$

$$
\theta_{\chi}(A \circ z)=\chi(d) j(A, z) \theta_{\chi}(z),
$$

where

$$
j(A, z)= \begin{cases}\varepsilon_{d}^{-1} \chi_{c}(d) N(c z+d)^{1 / 2} & \text { if } c \neq 0, \\ 1 & \text { if } c=0,\end{cases}
$$

with the following additional definitions. $N(z)^{1 / 2}=\sqrt{z \bar{z}}=|z|$ with $\bar{z}=$ $\bar{x}-k y . \chi_{c}(d)$ is the Kronecker symbol associated with the field extension $K(\sqrt{c}) / K . \varepsilon_{d}$ is periodic modulo 4 and if $d$ is a first degree prime, then

$$
\varepsilon_{d}=\left(\frac{t_{0} \bar{d}}{d}\right) \underline{\varepsilon}_{d \bar{d}}
$$

where

$$
\underline{\varepsilon}_{d \bar{d}}= \begin{cases}1 & \text { if } d \bar{d} \equiv 1(\bmod 4), \\ i & \text { if } d \bar{d} \equiv 3(\bmod 4)\end{cases}
$$

is from the rational case. The other factor is a Legendre symbol. By Dirichlet's theorem on primes in an arithmetic progression, this definition is sufficient to define $\varepsilon_{d}$ for all $d$ relatively prime to 4 , which is in any case a necessary condition for $A$ to be in $\Gamma_{0}(N)$. The periodicity of $\varepsilon_{d}$ also allows us to say that $\varepsilon_{d}$ makes sense for non-integral $d$ which have numerator and denominator relatively prime to 2 .

We will consider the functions $f$ defined on $\mathbb{H}$ that satisfy

$$
f(A \circ z)=\chi(d) j(A, z) f(z)
$$

for all $A$ in $\Gamma_{0}(N)$. As $\left(\begin{array}{ll}1 & b \\ 0 & 1\end{array}\right) \in \Gamma_{0}(N)$ every such $f$ has $f(z+b)=f(z)$ and hence has a Fourier expansion $\sum_{b \in O_{K}} a(b) q(b)$. If there exists a $D$ 
such that $D a(b)$ is an integer for all $b$ then we say $f$ is a modular form in $\boldsymbol{M}_{0}\left(N, \frac{1}{2}, \chi\right)$. In other words, the space of modular forms $\boldsymbol{M}_{0}\left(N, \frac{1}{2}, \chi\right)$ that we are considering has a basis of forms with Fourier coefficients from a number field with a bounded denominator. That this $D$ always exists in the rational case is proven in [3] by reducing to the analogous situation for the integer weight case, which is proven in [4].

The set of $f \in M_{0}\left(N, \frac{1}{2}, \chi\right)$ that are zero as $z$ approaches $x+\infty k$ or the other cusps $x+0 k$ with $x$ in our number field $K$ will be called cusp forms and denoted by $\boldsymbol{S}_{0}\left(N, \frac{1}{2}, \chi\right)$.

Let $r(\psi)$ be the conductor, or minimum period, of a character $\psi$. The conductor is only unique up to multiplication by a unit. This slight ambiguity will not be consequential. $\psi$ is primitive if $r(\psi)$ is the same as $\psi$ 's period of definition. Also, $\psi$ is even if $\psi(-1)=1$ and odd otherwise. A modular form associated with an odd character is identically zero, so we need only consider even characters.

Let $\Omega(N, \chi)$ be the set of ordered pairs $(\psi, t)$ with $\psi$ an even primitive character, $t \in O_{K}$ with only a single $t$ allowed from among the set $t$, $u t$, and $u^{\prime} t$ for each $\psi$, where $u$ and $u^{\prime}$ are square units in the field $K$, and

(i) $4 r^{2}(\psi) t \mid N$,

(ii) $\chi(b)=\psi(b) \chi_{t}(b)$ for all $b$ prime to $N$.

Condition (ii) can be looked at in two ways. First, it says that $\psi$ is the primitive character associated with $\chi \chi_{t}$. Second, it says that $\chi$ is determined by $\psi$ and $t$. Note that the extra conditions on $t$ only apply if $K=\mathbb{Q}(\sqrt{-1})$ or $\mathbb{Q}(\sqrt{-3})$, the fields which have extra units in their integer rings.

If $\psi$ has conductor $r, \psi$ can be uniquely factored

$$
\psi=\prod_{\pi \mid r} \psi_{\pi}
$$

where the conductor of $\psi_{\pi}$ is the highest power of $\pi$ that divides $r$. $\psi$ is called totally even if all the $\psi_{\pi}$ 's are even. A totally even character is always the square of another character with period $r$ or $2 r$, depending on if $r$ is an odd or an even number respectively.

Define $\Omega_{e}(N, \chi)$ to be the pairs $(\psi, t)$ in $\Omega(N, \chi)$ with $\psi$ totally even and $\Omega_{c}(N, \chi)$ to be the rest of $\Omega(N, \chi)$.

The shifted theta functions are defined for $t \in O_{K}$ as

$$
\theta_{\psi, t}=\sum_{b \in O_{K}} \chi(b) q\left(t b^{2}\right) \text {. }
$$

We are now ready to state the generalized Serre-Stark theorems.

Theorem A. $\boldsymbol{M}_{0}\left(N, \frac{1}{2}, \chi\right)$ has the following basis:

$$
\left\{\theta_{\psi, t} \mid(\psi, t) \in \Omega(N, \chi)\right\} .
$$


Theorem B. $\boldsymbol{S}_{0}\left(N, \frac{1}{2}, \chi\right)$ has the following basis:

$$
\left\{\theta_{\psi, t} \mid(\psi, t) \in \Omega_{c}(N, \chi)\right\} .
$$

2. Operators. The main tool in the proofs of Theorems A and B is the use of linear operators that act on the spaces of modular forms. To define these operators we first extend the group $S L_{2}(\mathbb{C})$ to a group $\mathfrak{G}$ whose action resembles that of a multiplier system:

$$
\mathfrak{G}=\left\{M, \phi(z) \mid M \in S L_{2}(\mathbb{C}), \phi(z): \mathbb{H} \rightarrow \mathbb{C}\right\}
$$

and the group action is

$$
\{M, \phi(z)\}\{N, \psi(z)\}=\{M N, \phi(N \circ z) \psi(z)\} .
$$

If $A \in \Gamma_{0}(N)$, let $A^{*}=\{A, j(A, z)\} \in \mathfrak{G}$.

For $\{M, \phi(z)\} \in \mathfrak{G}$, define the slash operator

$$
f \mid\{M, \phi(z)\}=\phi^{-1}(z) f(M \circ z) .
$$

Thus, if $f \in M_{0}\left(N, \frac{1}{2}, \chi\right)$ and $A \in \Gamma_{0}(N)$, then

$$
f \mid A^{*}=\chi(d) f .
$$

Almost all of the operators we will need are either elements of $\mathfrak{G}$ or linear combinations of them. Elements of $\mathfrak{G}$ acting by the slash operator can be combined in the following manner:

$$
f\left|\sum c_{i}\left\{M_{i}, \phi_{i}(z)\right\}=\sum c_{i} f\right|\left\{M_{i}, \phi_{i}\right\} .
$$

The most important operators are the Hecke operators, defined below for any first degree prime $\pi$ :

$$
\begin{aligned}
T\left(\pi^{2}\right)= & |\pi|^{-3}\left[\sum_{j \in O /\left(\pi^{2}\right)}\left\{\left(\begin{array}{cc}
\pi^{-1} & j \pi^{-1} \\
0 & \pi
\end{array}\right),|\pi|\right\}\right. \\
& +\chi(\pi) \sum_{\substack{j \in O /(\pi) \\
j \neq 0}}\left\{\left(\begin{array}{cc}
1 & j \pi^{-1} \\
0 & 1
\end{array}\right), \varepsilon_{\pi}^{-1} \chi_{-j}(\pi)\right\} \\
& \left.+\chi\left(\pi^{2}\right)\left\{\left(\begin{array}{cc}
\pi & 0 \\
0 & \pi^{-1}
\end{array}\right),|\pi|^{-1}\right\}\right] \quad \text { if } \pi \nmid N
\end{aligned}
$$

and

$$
T\left(\pi^{2}\right)=|\pi|^{-3} \sum_{j \in O /\left(\pi^{2}\right)}\left\{\left(\begin{array}{cc}
\pi^{-1} & j \pi^{-1} \\
0 & \pi
\end{array}\right),|\pi|\right\} \quad \text { if } \pi \mid N .
$$

Finally, if $4 \pi \mid N$, then

$$
T(\pi)=|\pi|^{-3 / 2} \sum_{j(\bmod \pi)}\left\{\left(\begin{array}{cc}
\pi^{-1 / 2} & j \pi^{-1 / 2} \\
0 & \pi^{1 / 2}
\end{array}\right),|\pi|^{1 / 2}\right\} .
$$


These are well-defined as exchanging $j^{\prime}$ for $j$, with $j \equiv j^{\prime}\left(\bmod \pi^{2}\right)$, or $(\bmod ) \pi$, in any sum would merely be changing the matrix by a translation, i.e. multiplying on the left by $\left(\begin{array}{ll}1 & l \\ 0 & 1\end{array}\right)$ for some integer $l$.

Theorem 1. For $f \in \boldsymbol{M}_{0}\left(N, \frac{1}{2}, \chi\right), f \mid T\left(\pi^{2}\right) \in \boldsymbol{M}_{0}\left(N, \frac{1}{2}, \chi\right)$ and if $4 \pi \mid N$ then $f \mid T(\pi) \in M_{0}\left(N, \frac{1}{2}, \chi \chi_{\pi}\right)$. If $f(z)=\sum_{b \in O_{K}} a(b) q(b)$, then $f \mid T\left(\pi^{2}\right)=$ $\sum_{b \in O_{K}} c(b) q(b)$ where

$$
c(b)= \begin{cases}a\left(b \pi^{2}\right)+|\pi|^{-2} \chi(\pi)\left(\frac{b}{\pi}\right) a(b)+|\pi|^{-2} \chi\left(\pi^{2}\right) a\left(b / \pi^{2}\right) & \text { if } \pi \nmid N, \\ a\left(b \pi^{2}\right) & \text { if } \pi \mid N .\end{cases}
$$

If $4 \pi \mid N$ then $f \mid T(\pi)=\sum_{b \in O_{K}} c(b) q(b)$ where $c(b)=a(b \pi)$. Furthermore, any two of these operators commute.

The proof is a several pages long exercise. We provide the following outline. Let $A=\left(\begin{array}{ll}a & b \\ c & d\end{array}\right) \in \Gamma_{0}(N)$. First, we need $f\left|T\left(\pi^{2}\right)\right| A^{*}=\chi(d) f \mid T\left(\pi^{2}\right)$. For any $T \in \mathfrak{G}$ that is part of the Hecke operator, there exists $T^{\prime}$ part of the same operator and an $R \in \Gamma_{0}(N)$ such that $T A^{*}=\gamma R^{*} T^{\prime}$, where $\gamma$ is a root of unity that exactly compensates for the cases when $T$ and $T^{\prime}$ come from different summations of the Hecke operator. We may assume that $d$ is a first degree prime, since after it is proven in that case, the general case follows by multiplying by a translation matrix. In addition to the fact that whenever Legendre, Jacobi, and Kronecker symbols coincide they are equal, the following two lemmas are useful for verifying this theorem.

Lemma 1. For $(\pi, d)=(\pi, 4)=(d, 4)=1$,

$$
\frac{\varepsilon_{\pi d}}{\varepsilon_{\pi} \varepsilon_{d}}=\left(\frac{d}{\pi}\right)\left(\frac{\pi}{d}\right) \text {. }
$$

Proof. Take

$$
A=\left(\begin{array}{cc}
a & b \\
\pi N & d
\end{array}\right), \quad B=\left(\begin{array}{cc}
e & f \\
d N & \pi
\end{array}\right) .
$$

The lemma follows from the fact that $\theta\left|A^{*}\right| B^{*}=\theta \mid(A B)^{*}$.

By the quadratic reciprocity law, $\left(\frac{d}{\pi}\right)\left(\frac{\pi}{d}\right)$ equals a quadratic character depending on $\pi$ evaluated at $d$ (or vice versa) of period 4 . Call this $\chi_{4 \pi}(d)$. By letting $d=\pi-4$, we see that

$$
1=\left(\frac{4}{\pi-4}\right)=\left(\frac{\pi}{\pi-4}\right)=\chi_{4 \pi}(\pi-4)\left(\frac{\pi-4}{\pi}\right)=\chi_{4 \pi}(\pi)\left(\frac{-1}{\pi}\right) .
$$

And thus we have as a corollary that $\chi_{4 \pi}(\pi)=\left(\frac{-1}{\pi}\right)$, so that we can also say

$$
\frac{\varepsilon_{\pi d}}{\varepsilon_{\pi} \varepsilon_{d}}=\chi_{4 \pi}(\pi d)\left(\frac{-1}{\pi}\right)
$$


Lemma 2. If $K \neq \mathbb{Q}(\sqrt{-1})$ then $\varepsilon_{\pi} \neq-1$.

Proof. We may assume $\pi$ is a first degree prime. Suppose $\pi$ lies over a rational prime $p$. If $p \equiv 3(\bmod 4)$ then $e_{\pi}= \pm i$, by the definition of $\varepsilon_{\pi}$. Thus $p \equiv 1(\bmod 4)$, and $\varepsilon_{\pi}=\left(\frac{t_{0} \bar{\pi}}{\pi}\right)$.

Let $K=\mathbb{Q}(\sqrt{d})$ and $d \equiv 1(\bmod 4)$ and $\pi=(a+b \sqrt{d}) / 2$ with $a \equiv b$ $(\bmod 2)$. Choose $t_{0}=1 / \sqrt{d}$ (the sign of $t_{0}$ is irrelevant). Now

$$
\varepsilon_{\pi}=\left(\frac{\left(\frac{a-b \sqrt{d}}{2}\right) \frac{1}{\sqrt{d}}}{\frac{a+b \sqrt{d}}{2}}\right)=\left(\frac{b}{p}\right)=1 .
$$

The case of $K=\mathbb{Q}(\sqrt{-2})$ has only a minor variation.

If $K=\mathbb{Q}(\sqrt{-1})$ and we make the further restriction that $\pi=a+b i$ lies over $p \equiv 1(\bmod 8)$, then in a similar manner, $\varepsilon_{\pi}=1$. For more details, see the appendix.

These two lemmas combine to give an evaluation of $\varepsilon_{d}$ that is particularly useful. We specialize the values in the first lemma to get

$$
\frac{\varepsilon_{d \pi^{-1}} \varepsilon_{d \pi}}{\varepsilon_{d^{2}}}=\chi_{4 d \pi}\left(d^{2}\right)\left(\frac{-1}{d \pi}\right)=\left(\frac{-1}{d \pi}\right) .
$$

And the second lemma tells us that $\varepsilon_{d^{2}}=1$ since $d^{2}$ always lies over a number congruent to $1(\bmod 8)$. Thus

$$
\varepsilon_{d \pi^{-1}}=\varepsilon_{d \pi}^{-1}\left(\frac{-1}{d \pi}\right)=\varepsilon_{d \pi} .
$$

We now return to the outline of the proof of Theorem 1. To find the Fourier coefficients, recall that

$$
f(z)=\sum_{b \in O_{K}} a(b) q(b)
$$

where

$$
q(b)=\exp \left\{2 \pi i \operatorname{tr}\left(t_{0} x b+k\left|t_{0} y b\right|\right)\right\}:=e\left\{t_{0} x b+k\left|t_{0} y b\right|\right\} .
$$

Now for $\pi \nmid N$ we have

$$
\begin{aligned}
|\pi|^{3} f \mid T\left(\pi^{2}\right)= & \sum_{j\left(\bmod \pi^{2}\right)}|\pi|^{-1} f\left(\left(\pi^{-1} z+j \pi^{-1}\right) \pi^{-1}\right) \quad\left(=\Sigma_{1}\right) \\
& +\chi(\pi) \sum_{\substack{j(\bmod \pi) \\
j \neq 0}} \varepsilon_{\pi} \chi_{-j}(\pi) f\left(z+j \pi^{-1}\right) \quad\left(=\Sigma_{2}\right) \\
& +\chi\left(\pi^{2}\right)|\pi| f(\pi z \pi) .
\end{aligned}
$$


We simplify each summation in turn:

$$
\begin{aligned}
\Sigma_{1} & =\sum_{j\left(\bmod \pi^{2}\right)}|\pi|^{-1} \sum_{b \in O_{K}} a(b) e\left\{t_{0}\left(\frac{x+j}{\pi^{2}}\right) b+k\left|\frac{t_{0} y b}{\pi^{2}}\right|\right\} \\
& =|\pi|^{-1} \sum_{b \in O_{K}} a(b) e\left\{\frac{t_{0} x b}{\pi^{2}}+k\left|\frac{t_{0} y b}{\pi^{2}}\right|\right\} \sum_{j} e\left\{\frac{t_{0} j b}{\pi^{2}}\right\} .
\end{aligned}
$$

The sum on $j$ is zero unless $\pi^{2} \mid b$. After relabeling, we have

$$
\Sigma_{1}=|\pi|^{3} \sum_{b \in O_{K}} a\left(b \pi^{2}\right) q(b) .
$$

The next sum is

$$
\Sigma_{2}=\chi(\pi) \varepsilon_{p} \sum_{b \in O_{K}} a(b) q(b) \sum_{\substack{j(\bmod \pi) \\ j \neq 0}} e\left\{\frac{b t_{0} j}{\pi}\right\}\left(\frac{-j}{\pi}\right) .
$$

The inner sum is a Gauss sum that is zero if $\pi \mid b$ and otherwise is equal to $\underline{\varepsilon}_{p}\left(\frac{-b t_{0} \bar{\pi}}{\pi}\right)|\pi|$, where $p$ is the rational prime under $\pi$ and $\underline{\varepsilon}$ is the $\varepsilon$ from the rational case (i.e. 1 if $p \equiv 1(\bmod 4)$ and $i$ if $p \equiv 3(\bmod 4))$. Thus

$$
\Sigma_{2}=|\pi| \chi(\pi) \sum_{b \in O_{K}}\left(\frac{b}{\pi}\right) a(b) q(b) .
$$

Lastly,

$$
\begin{aligned}
\chi\left(\pi^{2}\right)|\pi| f(\pi z \pi) & =|\pi| \chi\left(\pi^{2}\right) \sum_{b \in O_{K}} a(b) e\left\{t_{0} \pi^{2} x b+k\left|t_{0} \pi^{2} y b\right|\right\} \\
& =|\pi| \chi\left(\pi^{2}\right) \sum_{b \in O_{K}} a\left(b / \pi^{2}\right) q(b),
\end{aligned}
$$

where $a\left(\frac{b}{\pi^{2}}\right)=0$ if $\pi^{2} \nmid b$. Putting the above components together gives the result for $c(b)$. If $\pi \mid N$, then we just use $\Sigma_{1}$ to get the desired result. For $T(\pi)$, the argument is the same as for $T\left(\pi^{2}\right)$ when $\pi \mid N$.

The commutativity results follow from an examination of the Fourier coefficients.

The $T\left(\pi^{2}\right)$ operator preserves $\boldsymbol{M}_{0}\left(N, \frac{1}{2}, \chi\right)$. We also need some operators that send one space of modular forms to another. Let $f(z)=\sum_{b \in O_{K}} a(b) q(b)$.

The shift operator

$$
V(m)=|m|^{-1 / 2}\left\{\left(\begin{array}{cc}
m^{1 / 2} & 0 \\
0 & m^{-1 / 2}
\end{array}\right),|m|^{-1 / 2}\right\}
$$

acts by

$$
f \mid V(m)(z)=f\left(m^{1 / 2} z m^{1 / 2}\right)=\sum_{b \in O_{K}} a(b) q(m b) .
$$


The symmetry operator

$$
W(N)=\left\{\left(\begin{array}{cc}
0 & -N^{-1 / 2} \\
N^{1 / 2} & 0
\end{array}\right),\left|N^{1 / 2} z\right|\right\}
$$

acts by

$$
\begin{aligned}
f \mid W(N)(z) & =\left|N^{1 / 2} z\right|^{-1} f\left(-N^{-1 / 2}\left(N^{1 / 2} z\right)^{-1}\right) \\
& =\left|N^{1 / 2} z\right|^{-1} \sum_{b \in O_{K}} a(b) q\left(b N^{-1},-z^{-1}\right)
\end{aligned}
$$

and $f|W(N)| W(N)=f$.

The conjugation operator $H$ is defined by

$$
f \mid H(z)=\sum_{b \in O_{K}} \overline{a(b)} q(b) .
$$

Theorem 2. The operators $V(m), W(N)$, and $H$ send $\boldsymbol{M}_{0}\left(N, \frac{1}{2}, \chi\right)$ to $\boldsymbol{M}_{0}\left(N m, \frac{1}{2}, \chi \chi_{m}\right), \boldsymbol{M}_{0}\left(N, \frac{1}{2}, \bar{\chi} \chi_{N}\right)$, and $\boldsymbol{M}_{0}\left(N, \frac{1}{2}, \bar{\chi}\right)$ respectively. If $f \in$ $\boldsymbol{M}_{0}\left(N, \frac{1}{2}, \chi\right)$, then

$$
\begin{gathered}
f|V(m)| T\left(\pi^{2}\right)=f\left|T\left(\pi^{2}\right)\right| V(m) \quad \text { when } \pi \nmid m, \\
f|W(N)| T\left(\pi^{2}\right)=\bar{\chi}\left(\pi^{2}\right) f\left|T\left(\pi^{2}\right)\right| W(N) \quad \text { when } \pi \nmid N, \\
f|H| T\left(\pi^{2}\right)=f\left|T\left(\pi^{2}\right)\right| H .
\end{gathered}
$$

Proof. Let $A=\left(\begin{array}{ll}a & b \\ c & d\end{array}\right) \in \Gamma_{0}(N m)$ and note

$$
f\left|V(m) A^{*}=f\right|\left\{\left(\begin{array}{cc}
a & m b \\
c m^{-1} & d
\end{array}\right), \chi_{m}(d) j(A, z)\right\} V(m)=\chi \chi_{m}(d) f \mid V(m) .
$$

For $A=\left(\begin{array}{ll}a & b \\ c & d\end{array}\right) \in \Gamma_{0}(N)$,

$$
\begin{aligned}
f|W(N)| A^{*} & =f \mid\left\{\left(\begin{array}{cc}
d & -N^{-1} c \\
-N b & a
\end{array}\right), \chi_{N}(a) j(A, z)\right\} W(N) \\
& =\bar{\chi} \chi_{N}(d) f \mid W(N)
\end{aligned}
$$

since

$$
\varepsilon_{d}^{-1}\left(\frac{c}{d}\right)=\chi_{N}(a) \varepsilon_{a}^{-1}\left(\frac{-N b}{d}\right)=\varepsilon_{a}^{-1}\left(\frac{(1-a d) / c}{a}\right)=\varepsilon_{a}^{-1}\left(\frac{c}{a}\right) .
$$

And $f|H| A^{*}=\overline{\chi(d)} f \mid H$ is clear.

The first and third commutativity results follow from an examination of their Fourier coefficients. The second commutativity result requires comparing the elements of $\mathfrak{G}$ on each side modulo $\Gamma_{0}(N)$. They can be paired up in such a way that each part of $T\left(\pi^{2}\right) W(N)$ agrees with some part of $W(N) T\left(\pi^{2}\right)$ up to multiplication on the left by an $A^{*}$ for some $A \in \Gamma_{0}(N)$. $A$ can be found explicitly, and in each case the lower right entry makes the 
coefficients come out correctly, as long as it is remembered that the character in the Hecke operator depends on the space to which the operator is being applied.

We will also need an operator to lower the level. First, we define one that only lowers the level. Then, using this operator, we define another one that "undoes" the shift operator.

If $4 \pi_{0} \mid N$, write $\Gamma_{0}\left(N / \pi_{0}\right)$ as a disjoint union of cosets modulo $\Gamma_{0}(N)$ :

$$
\Gamma_{0}\left(N / \pi_{0}\right)=\bigcup_{j=1}^{\mu} \Gamma_{0}(N) A_{j}
$$

where $\mu=\left[\Gamma_{0}\left(N / \pi_{0}\right): \Gamma_{0}(N)\right] . S^{\prime}(\chi)=S^{\prime}\left(\chi, N, \pi_{0}\right)$ is the trace operator defined on $\boldsymbol{M}_{0}\left(N, \frac{1}{2}, \chi\right)$ by

$$
S^{\prime}(\chi)=\sum_{j=1}^{\mu} \chi\left(a_{j}\right) A_{j}^{*}=\sum_{j=1}^{\mu} \overline{\chi\left(d_{j}\right)} A_{j}^{*}
$$

where $a_{j}$ and $d_{j}$ are the diagonal entries of $A_{j}$.

That $S^{\prime}(\chi)$ is well-defined, takes $\boldsymbol{M}_{0}\left(N, \frac{1}{2}, \chi\right)$ to $\boldsymbol{M}_{0}\left(N / \pi_{0}, \frac{1}{2}, \chi\right)$ whenever $\chi$ is definable $\left(\bmod N / \pi_{0}\right)$, and commutes with $T\left(\pi^{2}\right)$ has the same proof as in [3]. Clearly, if $f \in \boldsymbol{M}_{0}\left(N / \pi_{0}, \frac{1}{2}, \chi\right)$ then

$$
f \mid S^{\prime}(\chi)=\sum_{j=1}^{\mu} \overline{\chi\left(d_{j}\right)} \chi\left(d_{j}\right) f=\mu f .
$$

The trace operator $S(\chi)$

$$
S(\chi)=\frac{1}{\mu}\left|\pi_{0}\right|^{1 / 2} W(N) S^{\prime}\left(\bar{\chi} \chi_{N}\right) W\left(N / \pi_{0}\right)
$$

"undoes" the shift operator in the following sense.

Lemma 3. Let $\pi_{0}$ be a prime such that $4 \pi_{0} \mid N$ and $\chi \chi_{\pi_{0}}$ is definable $\left(\bmod N / \pi_{0}\right)$. Then:

(a) The operator $S\left(\chi, N, \pi_{0}\right)$ maps $\boldsymbol{M}_{0}\left(N, \frac{1}{2}, \chi\right)$ into $\boldsymbol{M}_{0}\left(N / \pi_{0}, \frac{1}{2}, \chi \chi_{\pi_{0}}\right)$.

(b) If $f$ belongs to $\boldsymbol{M}_{0}\left(N, \frac{1}{2}, \chi\right)$, and $\left(\pi_{0}, m\right)=1$ then

$$
f\left|S\left(\chi, N, \pi_{0}\right)=f\right| S\left(\chi, N m, \pi_{0}\right) .
$$

(c) $S(\chi)$ commutes with all $T\left(\pi^{2}\right)$ for $\pi \nmid N$.

(d) If $g \in \boldsymbol{M}_{0}\left(N / \pi_{0}, \frac{1}{2}, \chi \chi_{\pi_{0}}\right)$, then $g \mid V\left(\pi_{0}\right) \in \boldsymbol{M}_{0}\left(N, \frac{1}{2}, \chi\right)$ and

$$
g\left|V\left(\pi_{0}\right)\right| S\left(\chi, N, \pi_{0}\right)=g .
$$

(e) Let $\pi$ be a prime such that $4 \pi \mid N, \pi \neq \pi_{0}$, and $\chi \chi_{\pi}$ is definable $(\bmod N / \pi)$. If $g \in \boldsymbol{M}_{0}\left(N / \pi, \frac{1}{2}, \chi \chi_{\pi}\right)$, we have

$$
g|V(\pi)| S\left(\chi, N, \pi_{0}\right)=g\left|S\left(\chi \chi_{\pi}, N / \pi, \pi_{0}\right)\right| V(\pi) .
$$


Proof. (a) Theorem 2 and the above show that $S(\chi)$ sends $\boldsymbol{M}_{0}\left(N, \frac{1}{2}, \chi\right)$ to $\boldsymbol{M}_{0}\left(N / \pi_{0}, \frac{1}{2},\left(\overline{\bar{\chi} \chi_{N}}\right) \chi_{N / \pi_{0}}\right)$ and part (a) follows since

$$
\chi \bar{\chi}_{N} \chi_{N / \pi_{0}}=\chi \bar{\chi}_{N} \chi_{N} \chi_{\pi_{0}}=\chi \chi_{\pi_{0}} .
$$

(b) If $\left(\begin{array}{ll}a & b \\ c & d\end{array}\right) \in \Gamma_{0}\left(N m / \pi_{0}\right)$, with $\left(m, \pi_{0}\right)=1$, then

$$
W(N m) A^{*} W\left(N m / \pi_{0}\right)=W(N)\left(\begin{array}{cc}
a & b m \\
c / m & d
\end{array}\right)^{*} W\left(N / \pi_{0}\right)
$$

implies (b).

(c) This statement follows from previous commutativity results.

(d) We have

$$
g\left|V\left(\pi_{0}\right)\right| W(N)=|\pi|^{-1 / 2} g \mid W\left(N / \pi_{0}\right)
$$

which is in $\boldsymbol{M}_{0}\left(N, \frac{1}{2}, \bar{\chi} \chi_{N}\right)$ and is thus invariant under $\frac{1}{\mu} S^{\prime}\left(\bar{\chi} \chi_{N}\right)$ and is sent to $\left|\pi_{0}\right|^{-1 / 2} g$ by $W\left(N / \pi_{0}\right)$ which proves (d).

(e) We have $4 \pi \pi_{0} \mid N$ and $\chi \chi_{\pi_{0}} \chi_{\pi}$ is definable $\left(\bmod N / \pi \pi_{0}\right)$. Now

$$
\begin{aligned}
g|V(\pi)| S\left(\chi, N, \pi_{0}\right) & =\frac{1}{\mu}\left|\pi_{0}\right|^{1 / 2} g|V(\pi)| W(N) S^{\prime}\left(\bar{\chi} \chi_{N}\right) W\left(N / \pi_{0}\right) \\
& =\frac{1}{\mu}\left|\pi_{0} \pi\right|^{1 / 2} g \mid W(N / \pi) S^{\prime}\left(\bar{\chi} \chi_{N}\right) W\left(N / \pi_{0}\right) \\
& =\frac{1}{\mu}\left|\pi_{0}\right|^{1 / 2} g\left|W(N / \pi) S^{\prime}\left(\bar{\chi} \chi_{N}\right)\right||\pi|^{1 / 2} W\left(N / \pi \pi_{0}\right) V(\pi) \\
& =g\left|S\left(\chi \chi_{\pi}, N / \pi, \pi_{0}\right)\right| V(\pi) .
\end{aligned}
$$

If $\pi$ is an arbitrary prime we define

$$
K(\pi)=1-T(\pi, N \pi) V(\pi),
$$

where $T(\pi, N \pi)$ is the Hecke operator applied to the level $N \pi$. We immediately have:

Lemma 4. If $f=\sum_{b \in O_{K}} a(b) q(b) \in M_{0}\left(N, \frac{1}{2}, \chi\right)$ then $f \mid K(\pi) \in$ $\boldsymbol{M}_{0}\left(N \pi^{2}, \frac{1}{2}, \chi\right)$ and is equal to $\sum_{(b, \pi)=1} a(b) q(b)$. If $\pi^{\prime} \nmid N \pi$, then $T\left(\pi^{\prime 2}\right)$ and $K(\pi)$ commute.

Note that all the above operators take cusp forms to cusp forms, as can be seen from their Fourier expansions.

3. Newforms. Let $f$ be an eigenform of all but finitely many $T\left(\pi^{2}\right) . f$ is an oldform if there exists a prime $\pi$ dividing $N / 4$ such that either $\chi$ is definable $(\bmod N / \pi)$ and $f \in M_{0}\left(N / \pi, \frac{1}{2}, \chi\right)$ or $\chi \chi_{\pi}$ is definable $(\bmod N / \pi)$ and $f=g \mid V(\pi)$ for some $g \in \boldsymbol{M}_{0}\left(N / \pi, \frac{1}{2}, \chi \chi_{\pi}\right)$. The oldforms span a subspace of $\boldsymbol{M}_{0}\left(N, \frac{1}{2}, \chi\right)$ denoted by $\boldsymbol{M}_{0}^{\text {old }}\left(N, \frac{1}{2}, \chi\right)$. If $f$ is an eigenform of all 
but finitely many $T\left(\pi^{2}\right)$ and $f \notin \boldsymbol{M}_{0}^{\text {old }}\left(N, \frac{1}{2}, \chi\right)$ we say that $f$ is a newform of level $N$.

Lemma 5. The symmetry operator $W(N)$ and the conjugation operator $H$ take oldforms to oldforms and newforms to newforms.

Proof. Same as in [3].

Lemma 6. Let $h \in \boldsymbol{M}_{0}^{\text {old }}\left(N, \frac{1}{2}, \chi\right)$ be a non-zero eigenform of all but finitely many $T\left(\pi^{2}\right)$. Then there is a divisor $N_{1}$ of $N$ with $\left|N_{1}\right|<|N|$, a character $\psi$ definable $\left(\bmod N_{1}\right)$ and a newform $g$ in $\boldsymbol{M}_{0}\left(N_{1}, \frac{1}{2}, \psi\right)$ such that $g$ and $h$ have the same eigenvalues for all but finitely many $T\left(\pi^{2}\right)$.

Pro of. Same as in [3], except we use induction on $|N|$.

Lemma 7. Let $\pi$ be a prime and $f=\sum_{b \in O_{K}} a(b) q(b)$ be a non-zero element of $\boldsymbol{M}_{0}\left(N, \frac{1}{2}, \chi\right)$ such that $a(b)=0$ whenever $\pi \nmid b$. Then $\pi \mid N / 4$, $\chi \chi_{\pi}$ is definable $(\bmod N / \pi)$ and $f=g \mid V(\pi)$ with $g \in \boldsymbol{M}_{0}\left(N / \pi, \frac{1}{2}, \chi \chi_{\pi}\right)$.

Proof. Same as in [3] except, due to non-commutativity, put

$$
\begin{aligned}
g(z) & =f\left(\pi^{-1 / 2} z \pi^{-1 / 2}\right)=\sum_{b \in O_{K}} a(\pi b) q(b) \\
& =|\pi|^{1 / 2} f \mid\left\{\left(\begin{array}{cc}
\pi^{-1 / 2} & 0 \\
0 & \pi^{1 / 2}
\end{array}\right),|\pi|^{1 / 2}\right\}
\end{aligned}
$$

and similarly normalize the other operators.

Theorem 3. Let $m \neq 0 \in O_{K}$, and $f=\sum_{b \in O_{K}} a(b) q(b) \in M_{0}\left(N, \frac{1}{2}, \chi\right)$ such that $a(b)=0$ whenever $(b, m)=1$. Then

$$
f=\sum_{\pi} f_{\pi} \mid V(\pi) \quad \text { with } f_{\pi} \in M_{0}\left(N / \pi, \frac{1}{2}, \chi_{\pi}\right)
$$

with $\pi$ running through the set of primes such that $\pi|m, 4 \pi| N$, and $\chi_{\chi_{\pi}}$ is definable $(\bmod N / \pi)$.

Furthermore, if $f$ is a cusp form, the $f_{\pi}$ can also be chosen to be cusp forms. If $f$ is an eigenform of all but finitely many $T\left(\pi^{\prime 2}\right)$, then the $f_{\pi}$ can also be chosen to be eigenforms of all but finitely many $T\left(\pi^{\prime 2}\right)$ and have the same eigenvalues as $f$.

Proof. Also the same as in [3].

COROLlary. If the function $f$ of Theorem 3 is an eigenform of all but finitely many $T\left(\pi^{\prime 2}\right)$, then $f \in M_{0}^{\text {old }}\left(N, \frac{1}{2}, \chi\right)$.

Lemma 8. Let $f=\sum_{b \in O_{K}} a(b) q(b)$ be a non-zero element of $\boldsymbol{M}_{0}\left(N, \frac{1}{2}, \chi\right)$ and let $\pi$ be a prime with $\pi \nmid N$. Assume that $f \mid T\left(\pi^{2}\right)=c_{\pi} f$ with $c_{\pi} \in \mathbb{C}$. Let $b \neq 0$ and $\pi^{2} \nmid b$. Then:

(a) $a\left(b \pi^{2 n}\right)=a(b) \chi(\pi)^{n}\left(\frac{b}{\pi}\right)^{n}$ for all $n>0$. 
(b) If $a(b) \neq 0$, then $\pi \nmid b$ and $c_{\pi}=\chi(\pi)\left(\frac{b}{\pi}\right)\left(1+|\pi|^{-2}\right)$.

Proof. Set

$$
A(T)=\sum_{n=0}^{\infty} a\left(b \pi^{2 n}\right) T^{n} .
$$

To put this in closed form, examine what happens after applying $T\left(\pi^{2}\right)$ to $f$. One obtains

$$
A(T)=a(b) \frac{1-|\pi|^{-2} \chi(\pi)\left(\frac{b}{\pi}\right) T}{1-c_{\pi} T+|\pi|^{-2} \chi(\pi)^{2} T^{2}} .
$$

Use $\pi$-adic analysis in the same way as in [3], Lemma 9.

Theorem 4. Let $f$ be as in Lemma 8 and let $N^{\prime}$ be a multiple of $N$. If $f \mid T\left(\pi^{2}\right)=c_{\pi} f$ for all $\pi \nmid N^{\prime}$, then there exists a square-free integer $t$, unique up to multiplication by a square unit, such that $a(b)=0$ if $b / t$ is not a square. Moreover, we have:

(a) $t \mid N^{\prime}$.

(b) $c_{\pi}=\chi(\pi)\left(\frac{t}{\pi}\right)\left(1+|\pi|^{-2}\right)$ if $\pi \nmid N^{\prime}$.

(c) $a\left(b u^{2}\right)=a(b) \chi(u)\left(\frac{t}{u}\right)$ if $\left(u, N^{\prime}\right)=1$, where $\left(\frac{*}{*}\right)$ is the Jacobi symbol.

Proof. Let $b$ and $b^{\prime}$ be two integers such that neither $a(b)$ nor $a\left(b^{\prime}\right)$ is zero. Let $P$ be the set of primes $\pi$ with $\pi \nmid N^{\prime} b b^{\prime}$. If $\pi \in P$, Lemma 8 shows that

$$
\chi(\pi)\left(\frac{b}{\pi}\right)\left(1+|\pi|^{-2}\right)=c_{\pi}=\chi(\pi)\left(\frac{b^{\prime}}{\pi}\right)\left(1+|\pi|^{-2}\right)
$$

hence

$$
\left(\frac{b}{\pi}\right)=\left(\frac{b^{\prime}}{\pi}\right)
$$

and thus $b / b^{\prime}$ is a square. We may write $b=t v^{2}, b^{\prime}=t v^{\prime 2}$ with $t$ square-free, and we have proven the existence of $t$. Write $v$ as $\pi^{n} u$ with $(\pi, u)=1$ for any $\pi \nmid N^{\prime}$. Since $t$ is square-free, we have $\pi^{2} \nmid t$ and we can apply Lemma 8 . This tells us that

$$
a(b)=a\left(t u^{2} \pi^{2 n}\right)=a\left(t u^{2}\right) \chi(\pi)^{n}\left(\frac{t u^{2}}{\pi}\right)^{n}=a\left(t u^{2}\right) \chi(\pi)^{n}\left(\frac{t}{\pi}\right)^{n} .
$$

Hence, $\pi \nmid t u^{2}$, which implies that $\pi \nmid t$. Thus, $\pi \nmid N^{\prime}$ implies $\pi \nmid t$. What we need is just the contrapositive of this statement, i.e. $\pi \mid t$ implies $\pi \mid N^{\prime}$. Since $t$ is square-free, this shows that $t \mid N^{\prime}$. Lastly,

$$
c_{\pi}=\chi(\pi)\left(\frac{t u^{2}}{\pi}\right)\left(1+|\pi|^{-2}\right)=\chi(\pi)\left(\frac{t}{\pi}\right)\left(1+|\pi|^{-2}\right) .
$$

Part (c) follows by factoring $u$ and applying Lemma 8 . 
4. Proof of results. Let $f=\sum_{b \in O_{K}} a(b) q(b)$ be a newform of level $N$ belonging to $\boldsymbol{M}_{0}\left(N, \frac{1}{2}, \chi\right)$.

Lemma 9. The $t$ of Theorem 4 is a unit and $a(t) \neq 0$.

Proof. Suppose $a(u)=0$ for all units $u$ of the field $K$. By Theorem 4, $a\left(u b^{2}\right)=a(u) \chi(b)\left(\frac{t}{b}\right)=0$ for all $b$ such that $\left(b, N^{\prime}\right)=1$. Theorem 3 then shows that $f$ must be an oldform, and we have our contradiction.

Before we can prove the next lemma, we must say something about the two cases when we have extra units. If $K=\mathbb{Q}(\sqrt{-1})$, then

$$
f|V(-1)=f|\left\{\left(\begin{array}{cc}
-i & 0 \\
0 & i
\end{array}\right), 1\right\}=f(-x+y k)=\sum_{b \in O_{K}} a(-b) q(b)=\chi(i) f .
$$

Thus, $a(b)=\chi(i) a(-b)$. But $1=\chi(-1)=\chi\left(i^{2}\right)=\chi(i)^{2}$, and we have $\chi(i)= \pm 1$, so that $a(b)= \pm a(-b)$. Similarly, if $k=\mathbb{Q}(\sqrt{-3})$ and $\varrho=$ $(-1+\sqrt{-3}) / 2$, then $a(\varrho b)=a(b)$ or $\varrho a(b)$ or $\varrho^{2} a(b)$. In either field, the important thing is that $a(b)$ and $a(u b)$ are related in the same way for every form in a given space for any given unit $u$.

LEMma 10. If $g \in \boldsymbol{M}_{0}\left(N, \frac{1}{2}, \chi\right)$ is an eigenform of all but finitely many $T\left(\pi^{2}\right)$ with the same eigenvalues as $f$, then $g$ is a multiple of $f$.

Proof. Call the unit of Lemma $9 u$ and normalize $f$ so that $a(u)=1$. Let $w$ be any non-square unit in $K$. The coefficient of $q(u w)$ in $g$ must be zero, since otherwise $f+g \in \boldsymbol{M}_{0}\left(N, \frac{1}{2}, \chi\right)$, which would have non-zero coefficients on both the $q(u)$ and $q(u w)$ terms, which contradicts the conditions we have on $u$ from Theorem 4 . Let $c$ be the coefficient of $q(u)$ in $g$ and let $h=g-c f$. In $h$, the coefficient of $q(v)$ is zero for all units $v \in k$ since the coefficients of $q(v)$ have the same relationships in $g$ as in $f$. Assume $h \neq 0$. $h$ is obviously an eigenform with the same eigenvalues as $f$. By Lemma 9, $h$ is not a newform. Therefore, $h \in \boldsymbol{M}_{0}^{\text {old }}\left(N, \frac{1}{2}, \chi\right)$. By Lemma 6 , there exist a divisor $N_{1}$ of $N$ with $\left|N_{1}\right|<|N|$, a character $\psi$ defined $\left(\bmod N_{1}\right)$, and a newform $h_{1} \in \boldsymbol{M}_{0}\left(N, \frac{1}{2}, \psi\right)$ with the same eigenvalues as $h$ for all but finitely many $T\left(\pi^{2}\right)$. The eigenvalue $c_{\pi}$ is

$$
\chi(\pi)\left(\frac{u}{\pi}\right)\left(1+|\pi|^{-2}\right)=\psi(\pi)\left(\frac{u_{1}}{\pi}\right)\left(1+|\pi|^{-2}\right)
$$

which implies

$$
\chi(\pi)=\psi(\pi)\left(\frac{u u_{1}}{\pi}\right),
$$

where $u_{1}$ is the unit of $h_{1}$ given by Lemma 9 .

If $\left(\frac{u}{\pi}\right)=\left(\frac{u_{1}}{\pi}\right)$, then $\chi=\psi$ and we have $h_{1} \in M_{0}^{\text {old }}\left(N, \frac{1}{2}, \chi\right)$. Otherwise, let $h_{2}=h_{1} \mid V\left(u u_{1}\right) \in \boldsymbol{M}_{0}\left(N_{1}, \frac{1}{2}, \psi \chi_{u u_{1}}\right)$. Therefore, $h_{2} \in \boldsymbol{M}_{0}^{\text {old }}\left(N, \frac{1}{2}, \chi\right)$. Let $d$ be the coefficient of $q(u)$ in $h_{i}(i=1$ or 2 -whichever one is in 
$\left.\boldsymbol{M}_{0}^{\text {old }}\left(N, \frac{1}{2}, \chi\right)\right) . \quad d \neq 0$ since $h_{1}$ is a newform. As before, $f-\frac{1}{d} h_{i}$ is an oldform. But

$$
f=\frac{1}{d} h_{i}+\left(f-\frac{1}{d} h_{i}\right)
$$

is a sum of two forms in $\boldsymbol{M}_{0}^{\text {old }}\left(N, \frac{1}{2}, \chi\right)$. But $f$ is a newform. Therefore our assumption that $h \neq 0$ must be false and $g=c f$.

Lemma 11. $f$ is an eigenform of every $T\left(\pi^{2}\right)$. If $4 \pi \mid N$, then $f \mid T\left(\pi^{2}\right)$ $=0$.

Proof. Let $g=f \mid T\left(\pi^{2}\right)$ for any $\pi$. Theorem 1 gives us that $g \mid T\left(\pi^{\prime 2}\right)=$ $f\left|T\left(\pi^{\prime 2}\right)\right| T\left(\pi^{2}\right)=c_{\pi^{\prime}} g$ for all but finitely many $\pi^{\prime}$. Therefore, by Lemma 10 , $g$ is a multiple of $f$, i.e. $f$ is an eigenform of $T\left(\pi^{2}\right)$ for all $\pi$. If $4 \pi \mid N$, we again use Theorem 1.

$$
f \mid T(\pi)=\sum_{b \in O_{K}} a(b \pi) q(b) \in M_{0}\left(N, \frac{1}{2}, \chi \chi_{\pi}\right)
$$

but $a(b \pi) \neq 0$ only if $b$ is of the form $b=u c^{2} \pi$. Now

$$
\begin{aligned}
f \mid T(\pi) & =\sum_{b \in O_{K}} a\left(u c^{2} \pi^{2}\right) q\left(u c^{2} \pi\right) \\
& =f\left|T\left(\pi^{2}\right)\right| V(\pi)=c_{\pi} f \mid V(\pi) .
\end{aligned}
$$

If $c_{\pi} \neq 0$, Lemma 7 shows that $\chi$ is definable $(\bmod N / \pi)$ and $f \mid T(\pi)=$ $g \mid V(\pi)$ for some $g \in M_{0}\left(N / \pi, \frac{1}{2}, \chi\right)$. Thus

$$
c_{\pi} f|V(\pi)=g| V(\pi)
$$

and by applying the trace operator we have $c_{\pi} f=g$. Therefore, $f \in$ $\boldsymbol{M}_{0}\left(N / \pi, \frac{1}{2}, \chi\right)$, which is impossible since $f$ is a newform. Thus $c_{\pi}=0$.

Note that this implies $a\left(b^{2}\right)=0$ if $(b, N) \neq 1$.

Lemma 12. The level $N$ of the newform $f$ is a square times a unit.

Proof. If $\pi \nmid N$, we have $f \mid T\left(\pi^{2}\right)=c_{\pi} f$ with $c_{\pi}=\chi(\pi)\left(\frac{u}{\pi}\right)\left(1+|\pi|^{-2}\right)$. By Theorem 2,

$$
f|W(N)| T\left(\pi^{2}\right)=\bar{\chi}(\pi)^{2} c_{\pi} f\left|W(N)=\overline{c_{\pi}} f\right| W(N)
$$

and

$$
f|H| T\left(\pi^{2}\right)=\left(c_{\pi} f\right) \mid H=\overline{c_{\pi}}(f \mid H)
$$

since $H$ is anti-linear.

Lemma 5 tells us that $f \mid W(N)$ and $f \mid H$ are both newforms of level $N$ and characters $\bar{\chi} \chi_{N}$ and $\bar{\chi}$ respectively. Furthermore, they have the same eigenvalues corresponding to $T\left(\pi^{2}\right)$ for $\pi \nmid N$. By Theorem 4 the eigenvalues determine the character up to $\chi_{u}(\pi)$ for some unit $u$. Thus $\chi_{N}=\chi_{u}$, whence $N$ is a square times a unit. 
TheOrem 5. If $f=\sum_{b \in O_{K}} a(b) q(b) \in M_{0}\left(N, \frac{1}{2}, \chi\right)$ is a newform with $a(1)=1$ and $r$ is the conductor of $\chi$, then $N=4 r^{2}$ and $f=\theta_{\chi}$.

Proof. Let $N_{1}$ and $r_{1}$ be the products of all the prime divisors of $N$ and $r$ to the first power. We have

$$
f(z)=\sum_{\left(b, N_{1}\right)=1} \chi(b) q\left(b^{2}\right) \quad \text { and } \quad \theta_{\chi}(z)=\sum_{\left(b, r_{1}\right)=1} \chi(b) q\left(b^{2}\right) .
$$

Since $r \mid N$, we see that $\left(b, N_{1}\right)=1$ implies $\left(b, r_{1}\right)=1$, and the difference between these two functions is

$$
f(z)=\theta_{\chi}-\sum_{\substack{\left(b, r_{1}\right)=1 \\\left(b, N_{1} / r_{1}\right) \neq 1}} \chi(b) q\left(b^{2}\right) .
$$

This function is in $\boldsymbol{M}_{0}\left(N^{\prime}, \frac{1}{2}, \chi\right)$, where $N^{\prime}$ is the least common multiple of $N$ and $4 r^{2}$. Let $c_{i}$ represent a product of $i$ primes, all of which divide $N_{1} / r_{1}$. Using the inclusion-exclusion principle, we obtain

$$
f(z)=\theta_{\chi}(z)+\sum_{i=1}^{l}(-1)^{i} \sum_{c_{i}} \chi\left(c_{i}\right) \theta_{\chi}\left(c_{i} z c_{i}\right),
$$

where $l$ is the number of prime factors of $N_{1} / r_{1}$. We know that $f \mid W(N) \in$ $\boldsymbol{M}_{0}\left(N, \frac{1}{2}, \bar{\chi} \chi_{N}\right)$ must have an integral $q$-expansion

$$
\begin{aligned}
\left.f|W(N)=| N^{1 / 2} z\right|^{-1} & {\left[\theta_{\chi}\left(-\left(N^{1 / 2} z N^{1 / 2}\right)^{-1}\right)\right.} \\
& \left.+\sum_{i=1}^{j}(-1)^{i} \sum_{c_{i}} \chi\left(c_{i}\right) \theta_{\chi}\left(-\left(c_{i}^{-1} N^{1 / 2} z N^{1 / 2} c_{i}^{-1}\right)^{-1}\right)\right]
\end{aligned}
$$

and using the theta inversion formula from [5], this is

$$
\sum_{\left(b, r_{1}\right)=1}\left[C(\chi, b) q\left(\frac{b^{2} N}{4 r^{2}}\right)+\sum_{c_{i}} C\left(\chi, b, c_{i}\right) q\left(\frac{b^{2} N}{4 r^{2} c_{i}^{2}}\right)\right]
$$

where the $C$ 's are constants that only depend on the arguments in the parentheses. Since the argument of $q$ must be integral, $4 r^{2} c_{i}^{2}$ divides $b^{2} N$. In particular, by the conditions on $b$, we have $4 r^{2} \mid N$. Thus, $N^{\prime}=N$ and $\theta_{\chi}-f$ is of level $N$. But what is important is that this says that $f$ is a linear combination of theta and shifted theta functions of a lower level, and thus is an oldform. The only resolution of this contradiction is for $N$ to equal $4 r^{2}$. Thus $f=\theta_{\chi}$.

Conversely, we have

THEOREM 6. If $\chi$ is an even primitive character of conductor $r$, then $\theta_{\chi}$ is a newform in $\boldsymbol{M}_{0}\left(4 r^{2}, \frac{1}{2}, \chi\right)$. 
Proof. Let $N=4 r^{2}$. From [5] we have $\theta_{\chi} \in \boldsymbol{M}_{0}\left(N, \frac{1}{2}, \chi\right)$, and by plugging into Theorem 1 , we see that $\theta_{\chi}$ is an eigenform of $T\left(\pi^{2}\right)$ with eigenvalue

$$
c_{\pi}=\left(1+|\pi|^{-2}\right) \chi(\pi) \quad \text { if } \pi \nmid N .
$$

Now assume $\theta_{\chi}$ is not a newform. Lemma 6 shows that we can find an $N_{1}$, with $\left|N_{1}\right|<|N|$ and $N_{1} \mid N$, and a character $\psi$ definable $\left(\bmod N_{1}\right)$, and a newform $f \in \boldsymbol{M}_{0}\left(N_{1}, \frac{1}{2}, \psi\right)$ such that $f$ and $\theta_{\chi}$ have the same eigenvalues for all but finitely $T\left(\pi^{2}\right)$, i.e.

$$
\left(1+|\pi|^{-2}\right) \psi(\pi)=\left(1+|\pi|^{-2}\right) \chi(\pi) \quad \text { for almost all } \pi .
$$

This shows that $\psi=\chi$. Theorem 5 now shows that $N_{1}=4 r^{2}$, which is a contradiction. Thus $\theta_{\chi}$ is a newform.

Theorem A. The theta functions $\theta_{\psi, t}$ with $(\psi, t) \in \Omega(N, \chi)$ form a basis for $\boldsymbol{M}_{0}\left(N, \frac{1}{2}, \chi\right)$.

Pr o of. First, we show that this set is linearly independent.

$\chi$ and $t$ determine $\psi$. Therefore, each $t$ only appears in a single pair of $\Omega(N, \chi)$. Suppose that

$$
\lambda_{1} \theta_{\psi_{1}, t_{1}}+\ldots+\lambda_{m} \theta_{\psi_{m}, t_{m}}=0, \quad \lambda_{i} \neq 0 \text { for all } i,
$$

with $\left|t_{i}\right| \leq\left|t_{i+1}\right|$, equality holding only if $t_{i}=u t_{i+1}$ for some non-square unit $u$. In any case, the coefficient of $q\left(t_{1}\right)$ in $\theta_{\psi_{1}, t_{1}}$ is non-zero and cannot be cancelled out by any of the other $\theta$-functions since none of them has a $q\left(t_{1}\right)$-term. Thus $\lambda_{1}=0$, a contradiction.

To show that the proposed basis generates $\boldsymbol{M}_{0}\left(N, \frac{1}{2}, \chi\right)$, we first prove

LEMma 13. There is a basis of $\boldsymbol{M}_{0}\left(N, \frac{1}{2}, \chi\right)$ containing only eigenforms for the $T\left(\pi^{2}\right)$, for $\pi \nmid N$.

Proof. Equivalently, we prove that the matrix representations of the $T\left(\pi^{2}\right)$ operators are simultaneously diagonalizable. Assume there is some $T\left(\pi^{2}\right)$ that is not diagonalizable. By putting it in its Jordan form, we can see that there is some element $g \in \boldsymbol{M}_{0}\left(N, \frac{1}{2}, \chi\right)$ such that

$$
g \mid U \neq 0 \quad \text { and } \quad g \mid U^{2}=0 \quad \text { where } U=T\left(\pi^{2}\right)-c_{\pi}
$$

for some eigenvalue $c_{\pi}$ of $T\left(\pi^{2}\right)$. If $g=\sum_{b \in O_{K}} d(b) q(b)$ we can assume that the $d(b)$ 's are algebraic with bounded denominator. Assume $b \nmid \pi$ and apply Theorem 1 to put

$$
D(T)=\sum_{m=0}^{\infty} d\left(b \pi^{2 m}\right) T^{m}
$$

into closed form (basically the same technique as in Lemma 8 but in this case a couple pages longer since we have $U^{2}$ instead of just $U$ ). We obtain

$$
D(T)\left[1-2 c_{\pi} T+\left(c_{\pi}^{2}+3 \chi\left(\pi^{2}\right)|\pi|^{-2}\right) T^{2}-2 c_{\pi} \chi\left(\pi^{2}\right)|\pi|^{-2} T^{3}+\chi\left(\pi^{4}\right)|\pi|^{-4} T^{4}\right]
$$




$$
\begin{array}{r}
=d(b)\left[1-2 c_{\pi} T-2 c_{\pi}\left(\frac{b}{\pi}\right) \chi(\pi)|\pi|^{-2} T^{2}-\chi\left(\pi^{3}\right)\left(\frac{b}{\pi}\right)|\pi|^{-4} T^{3}\right] \\
+d\left(b \pi^{2}\right)\left[T-\chi(\pi)|\pi|^{-2} T^{2}\right] .
\end{array}
$$

Now take $A(T)$ from Lemma 8, i.e.

$$
A(T)=\sum_{m=0}^{\infty} a\left(b \pi^{2 m}\right) T^{m}=\frac{a(b)}{1-\chi(\pi)\left(\frac{b}{\pi}\right) T},
$$

so that $a(b)=d(b)$. We know that $D(T)$ and $A(T)$ converge in the unit $\pi$-adic disc $\boldsymbol{U}$. By Lemma 8, the coefficients $a\left(b \pi^{2 m}\right)$ satisfy a recurrence relation that comes from our operator $U$. Hence, they also satisfy the same recursion relation as the $d\left(b \pi^{2 m}\right)$ since these come from $U^{2}$. Thus, since $a(b)=d(b), D(T)-A(T)$ is

$$
\frac{\left(d\left(b \pi^{2}\right)-a\left(b \pi^{2}\right)\right)\left(T-\chi(\pi)\left(\frac{b}{\pi}\right) T^{2}\right)}{1-2 c_{\pi} T+\left(c_{\pi}^{2}+3 \chi\left(\pi^{2}\right)|\pi|^{-2}\right) T^{2}-2 c_{\pi} \chi\left(\pi^{2}\right)|\pi|^{-2} T^{3}+\chi\left(\pi^{4}\right)|\pi|^{-4} T^{4}},
$$

which must be zero, since otherwise there would be poles in $\boldsymbol{U}$. Thus, $D(T)=A(T)$, from which $g \mid U=0$. Hence, $T\left(\pi^{2}\right)$ must be diagonalizable. Since the $T\left(\pi^{2}\right)$ commute, we have the lemma.

Now we can prove that the functions $\theta_{\psi, t}$ span $\boldsymbol{M}_{0}\left(N, \frac{1}{2}, \chi\right)$. By Lemma 13 , it suffices to show that any eigenform $f$ of all the $T\left(\pi^{2}\right)$, with $\pi \nmid N$, is a linear combination of $\theta_{\psi, t}$ with $(\psi, t) \in \Omega(N, \chi)$. We use induction on $|N|$. If $f$ is a newform, then Theorem 5 shows that $f$ is $\theta_{\psi, t}$, with $(\psi, t) \in \Omega(N, \chi)$. If $f$ is an oldform, then we have two cases. Either $f$ is an oldform of the first type and $\chi$ is definable $(\bmod N / \pi)$ and $f \in M_{0}\left(N / \pi, \frac{1}{2}, \chi\right)$, in which case, by induction, $f$ is a linear combination of $\theta_{\psi, t}$ with $(\psi, t) \in \Omega(N / \pi, \chi)$, which is a subset of $\Omega(N, \chi)$. Or else, $f$ is an oldform of the second type and $\chi \chi_{\pi}$ is definable $(\bmod N / \pi)$ and $f=g \mid V(\pi)$ with $g \in \boldsymbol{M}_{0}\left(N / \pi, \frac{1}{2}, \chi \chi_{\pi}\right)$. In this case, $g$ is a linear combination of $\theta_{\psi, t}$, with $(\psi, t) \in \Omega\left(N / \pi, \chi_{\pi}\right)$, which says that $f$ is a linear combination of $\theta_{\psi, t \pi}$ with $(\psi, t \pi) \in \Omega(N, \chi)$.

From [5], we know that $\theta_{\psi, t}$ is a cusp form if and only if $(\psi, t) \in \Omega_{c}(N, \chi)$. Now, we investigate whether or not linear combinations of $\theta_{\psi, t}$ with the $\psi$ 's all totally even can add up to a cusp form. First, we consider the case of a single fixed totally even $\psi$.

Lemma 14. If $\psi$ is a totally even character, $T$ is a finite set of integers, and

$$
f=\sum_{t \in T} \lambda_{t} \theta_{\psi, t}
$$

is a cusp form, then $f$ is identically zero.

Proof. Suppose $f \neq 0$. Note that $\theta_{\psi, t_{1}}$ and $\theta_{\psi, t_{2}}$ are in different spaces unless the quotient of $t_{1}$ and $t_{2}$ is a square. The common non-square factor 
is irrelevant, and we may assume all the $t$ 's are squares. Thus, we relabel, and say

$$
f=\sum_{t \in T} \lambda_{t} \theta_{\psi, t^{2}} .
$$

We wish to study $f$ at an arbitrary cusp $a / c$. The transformation formula given in [5] for $\theta$ tells us that if $\mathcal{D}$ is the different of $K$ and $\mu=(c) \mathcal{D}^{-1}$ then

$$
\begin{aligned}
f(z+a / c) & =\sum_{t \in T} \lambda_{t} \theta_{\psi, t^{2}}(z+a / c) \\
& =\sum_{t \in T} \lambda_{t} \sum_{n(\bmod \mu)} \psi(n) \exp \left\{2 \pi i \operatorname{tr}\left(t^{2} n^{2} a / c\right)\right\} \theta_{1}(t z t, n, \mu)
\end{aligned}
$$

where

$$
\theta_{1}(z, n, \mu)=\sum_{l \in \mu} e\left\{(l+n)^{2} x+i y|l+n|^{2}\right\} .
$$

By the inversion formula also developed in [5],

$$
\begin{aligned}
f\left(-z^{-1}+a / c\right)=\sum_{t \in T} \lambda_{t} \sum_{n(\bmod \mu)} \psi(n) & \exp \left\{2 \pi i \operatorname{tr}\left(t^{2} n^{2} a / c\right)\right\} \\
\times & N\left(2 \mathcal{D} \mu^{2}\right)|t z t| \theta_{1}^{\prime}\left(t z t, n,(2 \mathcal{D} \mu)^{-1}\right)
\end{aligned}
$$

where

$$
\theta_{1}^{\prime}\left(z, n,(2 \mathcal{D} \mu)^{-1}\right)=\sum_{l \in(2 \mathcal{D} \mu)^{-1}} e\left\{l^{2} x+i y|l|^{2}-2 \ln \right\}
$$

We can take the limit as $z$ goes to $x+k \infty$ and obtain the value of $f$ at $a / c$, which must be zero since $f$ is a cusp form. Thus

$$
\sum_{t \in T}|t|^{2} \lambda_{t} \sum_{n(\bmod \mu)} \psi(n) \exp \left\{2 \pi i \operatorname{tr}\left(t^{2} n^{2} a / c\right)\right\}=0 .
$$

Since we are only interested in reaching a contradiction, we will not have to evaluate this Gaussian sum explicitly. Toward this end, we introduce a periodic function $\alpha(n)(\bmod \nu)$ and its finite Fourier transform

$$
\widehat{\alpha}(m)=\frac{1}{\boldsymbol{N}(\nu)} \sum_{n(\bmod \nu)} \alpha(n) \exp \{2 \pi i \operatorname{tr}(-m n / \nu \mathcal{D})\} .
$$

The inverse transform is

$$
\alpha(n)=\sum_{m(\bmod \nu)} \widehat{\alpha}(m) \exp \{2 \pi i \operatorname{tr}(m n / \nu \mathcal{D})\} .
$$

Let $\nu$ be divisible by $2 \mu$ and all $t \in T$ and multiply $\widehat{\alpha}(a)$ by what we already have about $f$, which still holds if we change the modulus to $\nu$ :

$$
\widehat{\alpha}(a) \sum_{t \in T}|t|^{2} \lambda_{t} \sum_{n(\bmod \nu)} \psi(n) \exp \left\{2 \pi i \operatorname{tr}\left(t^{2} n^{2} a / c\right)\right\}=0 .
$$


Since this is true for all $a$ we can also write

$$
\sum_{a(\bmod \nu)} \widehat{\alpha}(a) \sum_{t \in T}|t|^{2} \lambda_{t} \sum_{n(\bmod \nu)} \psi(n) \exp \left\{2 \pi i \operatorname{tr}\left(t^{2} n^{2} a / c\right)\right\}=0 .
$$

Rearranging the terms gives

$$
\sum_{t \in T}|t|^{2} \lambda_{t} \sum_{n(\bmod \nu)} \psi(n) \sum_{a(\bmod \nu)} \widehat{\alpha}(a) \exp \left\{2 \pi i \operatorname{tr}\left(t^{2} n^{2} a / c\right)\right\}=0 .
$$

By the inverse Fourier transformation formula, this is

$$
\sum_{t \in T}|t|^{2} \lambda_{t} \sum_{n(\bmod \nu)} \psi(n) \alpha\left(t^{2} n^{2}\right)=0 .
$$

This is true for any function $\alpha$ defined modulo $\nu$. We now define a specific $\alpha$ that simplifies our expression.

Since $2 r(\psi) \mid \nu$ and $\psi$ is totally even, there exists a character $\varphi$ definable $(\bmod \nu)$ such that $\varphi^{2}=\psi($ actually definable $(\bmod 2 r(\psi))$ would suffice $)$. Pick $t_{0}$ such that $\left|t_{0}\right|<|t|$ for all $t$ such that $\lambda_{t} \neq 0$. Define

$$
\alpha(n)= \begin{cases}\varphi\left(n / t_{0}\right) & \text { if } t_{0} \mid n \text { and }\left(n / t_{0}, \nu\right)=1, \\ 0 & \text { otherwise. }\end{cases}
$$

Thus,

and in particular, if $t \neq t_{0}$

$$
\alpha\left(t_{0} n^{2}\right)= \begin{cases}\overline{\psi(n)} & \text { if }(n, \nu)=1 \\ 0 & \text { otherwise }\end{cases}
$$

$$
\alpha\left(t n^{2}\right)=0 .
$$

Thus, the above summation becomes

$$
\sum_{n(\bmod \nu)} \psi(n) \alpha\left(t_{0}^{2} n^{2}\right)=\sum_{n(\bmod \nu)} \psi(n) \bar{\psi}(n)
$$

which is greater than zero. Thus $f(z)=0$.

Now we consider the case where $\psi$ is not fixed, and we have our final result.

Theorem B. The theta functions $\theta_{\psi, t}$ with $(\psi, t) \in \Omega_{c}(N, \chi)$ form a basis for the space of cusp forms $\boldsymbol{S}_{0}\left(N, \frac{1}{2}, \chi\right)$.

Proof. In view of what we now know about the space $\boldsymbol{M}_{0}\left(N, \frac{1}{2}, \chi\right)$, this theorem is equivalent to the statement that no linear combination of $\theta_{\psi, t}$ with $(\psi, t) \in \Omega_{e}(N, \chi)$ is a cusp form. Suppose $f=\sum \lambda_{\psi, t} \theta_{\psi, t}$ is a cusp form. Let $V$ be the set of all linear combinations of $f \mid T\left(\pi^{2}\right)$ for all $\pi$. $V$ is a vector space that is invariant under the action $T\left(\pi^{2}\right)$. If $V$ is nonzero, then there is a form $g$ that is an eigenform of all the $T\left(\pi^{2}\right)$. Suppose $g(z)=\sum \lambda_{\psi, t}^{\prime} \theta_{\psi, t}$. But the eigenvalue of $\theta_{\psi, t}$ is $\left(1+|\pi|^{-2} \psi(\pi)\right)$, i.e. it 
changes with $\psi$. Thus $\lambda_{\psi, t}^{\prime}$ is non-zero only for a fixed $\psi$. But then the previous lemma says that $g$ is identically zero. Hence $V$ is zero, so $f$ is zero and we are done.

5. Appendix: Determination of the quadratic theta multiplier. The theta-multiplier defined by Stark in [5] simplifies in the real or imaginary quadratic case to

$$
j(A, z)= \begin{cases}\varepsilon_{d}^{-1} \chi_{c}(d) N(c z+d)^{1 / 2} & \text { if } c \neq 0, \\ 1 & \text { if } c=0\end{cases}
$$

with the same notation as in the introduction of this paper.

All the components of $j(A, z)$ are well understood except for the $\varepsilon_{d}$. The purpose of this appendix is to calculate $\varepsilon_{d}$ and to build a table of values that can be used when working with these functions. In particular, these values are needed in order to verify some of the theorems in Section 2 .

Recall from Section 1 that it is sufficient to consider (totally real) $d$ that generate first degree prime ideals. Thus we make a slight change of notation from the rest of the paper. Let $K=\mathbb{Q}(\sqrt{d}), d \in \mathbb{Z}$ square-free, $\pi \in O_{K}$ generates a first degree prime ideal over an odd rational prime $p$. With the new notation, we are trying to calculate $\varepsilon_{\pi}$, whose definition we now repeat:

$$
\varepsilon_{\pi}=\left(\frac{t \bar{\pi}}{\pi}\right) \underline{\varepsilon}_{p}
$$

where $t$ is a fixed generator of the inverse different of $K$ and

$$
\underline{\varepsilon}_{p}= \begin{cases}1 & \text { if } p \equiv 1(\bmod 4) \\ i & \text { if } p \equiv 3(\bmod 4)\end{cases}
$$

is from the rational case, where there are only two congruence classes relatively prime to 4 , and, by the above definition, each class takes a different value. In the quadratic case there are 4,8 , or 12 classes relatively prime to 4 depending on the field. From its definition one might expect that the values of $\varepsilon_{d}$ are equally distributed among \pm 1 or $\pm i$. We will show that this is not the case.

The first observation is that if $d \equiv 3(\bmod 4)$ then for some $a, b \in \mathbb{Z}$, $p=a^{2}-d b^{2} \equiv a^{2}+b^{2} \not \equiv 3(\bmod 4)$, which implies $\varepsilon_{\pi} \neq \pm i$. The most useful result is

Theorem 1. If $d<0, d \not \equiv 3(\bmod 4)$, then $\varepsilon_{\pi} \neq-1$.

Proof. We must show $\left(\frac{\bar{\pi} t}{\pi}\right)=1$ for all $\pi$ lying over $p \equiv 1(\bmod 4)$. If we make the additional assumption that $d \neq-3$, then there are only two possibilities for $t$ which differ by sign, the choice of which does not affect the Legendre symbol. 
If $d \equiv 1(\bmod 4)$, then

$$
\left(\frac{t}{\pi}\right)=\left(\frac{t^{-1}}{\pi}\right)=\left(\frac{\sqrt{d}}{\pi}\right)
$$

Setting $\pi=(a+b \sqrt{d}) / 2$, we have

$$
\left(\frac{\bar{\pi} t}{\pi}\right)=\left(\frac{\bar{\pi} \sqrt{d}-\pi \sqrt{d}}{\pi}\right)=\left(\frac{-b d}{\pi}\right) .
$$

Since there is only one real character on a group of $N(\pi)=p$ elements, this is the same as $\left(\frac{-b d}{p}\right)$. Letting $b=2^{l} b^{\prime}$ with $\left(2, b^{\prime}\right)=1$, we have

$$
\left(\frac{\bar{\pi} t}{\pi}\right)=\left(\frac{2^{l}}{p}\right)\left(\frac{b^{\prime} d}{p}\right)=\left(\frac{2^{l}}{p}\right)\left(\frac{p}{b^{\prime} d}\right)=\left(\frac{2^{l}}{p}\right)\left(\frac{a^{2}}{b^{\prime} d}\right)=\left(\frac{2^{l}}{p}\right) .
$$

If $l=0$ or 2 this is clearly 1 . If $l>2$ then $p \equiv a^{2} / 4 \equiv 1(\bmod 8)$, so $\left(\frac{2^{l}}{p}\right)=1$. Finally, if $l=1$, then $p \equiv a^{2} / 4-d b^{2} \equiv 3(\bmod 4)$, which contradicts $p \equiv 1$ $(\bmod 4)$.

If $d \equiv 2(\bmod 4)$, we let $\pi=a+b \sqrt{d}, p=a^{2}-d b^{2}$, and note that

$$
\left(\frac{t}{\pi}\right)=\left(\frac{2 \sqrt{d}}{\pi}\right)
$$

A similar computation gives

$$
\left(\frac{\bar{\pi} t}{\pi}\right)=\left(\frac{2^{l+1}}{p}\right)
$$

where again $l$ is the highest power of 2 dividing $b$. If $l=0$, then $p \equiv 3$ $(\bmod 4)$, the same contradiction as before. Thus, $l>0$ and $p \equiv a^{2} \equiv 1$ $(\bmod 8)$, so that we are done with this case.

Finally, if $d=-3$, the extra roots of unity give four additional possibilities for $t$. It suffices to show that $\left(\frac{( \pm 1 \pm \sqrt{-3}) / 2}{\pi}\right)=1$ for any $\pi$ lying over a rational prime congruent to $1(\bmod 4)$. We do this by noting that

$$
\left(\frac{(1+\sqrt{-3}) / 2}{(7+\sqrt{-3}) / 2}\right)=\left(\frac{-3}{13}\right)=1
$$

and that the other units are powers of this one so they are also squares modulo this prime. But the six associates of this prime cover all six residue classes $(\bmod 4)$ that have norm congruent to $1(\bmod 4)$. Thus the theorem is proved.

TheOREM 2. With the notation as in Theorem 1 , if $d \equiv 1$ or $2(\bmod 4)$, then for $\pi$ in half of the equivalence classes $\varepsilon_{\pi}=1$ and for the remaining classes $\varepsilon_{\pi}$ is equally often $i$ and $-i$. If $d \equiv 3(\bmod 4)$, then six of the eight values are 1 and two are -1 . 
Proof. Theorem 1 took care of the first claim. Consider $d \equiv 1$ or 2 and $p \equiv 3(\bmod 4)$. In calculating $\left(\frac{\bar{\pi} t}{\pi}\right)$, the only difference now is that the choice of $t$ determines the sign. So, with the same notation as before,

$$
\left(\frac{\bar{\pi} t}{\pi}\right)= \pm\left(\frac{b d}{p}\right)
$$

the sign depending only on which value of $t$ is chosen.

If $d \equiv 1(\bmod 4)$, this becomes

$$
\pm\left(\frac{2^{l}}{p}\right)\left(\frac{b^{\prime} d}{p}\right)= \begin{cases} \pm\left(\frac{2^{l}}{p}\right) & \text { if } b^{\prime} \equiv 1(\bmod 4), \\ \mp\left(\frac{2^{l}}{p}\right) & \text { if } b^{\prime} \equiv 3(\bmod 4) .\end{cases}
$$

But since $p \equiv 3(\bmod 4)$ we must have $l=0$ or 1 . Specializing further, if $d \equiv 1(\bmod 8)$, then $l=1$ and $\pi$ is congruent to either $\sqrt{d}$ or $2+\sqrt{d}$. In the first case, $b^{\prime} \equiv 1(\bmod 4)$ if and only if $p \equiv 7(\bmod 8)$. In the second case, $b^{\prime} \equiv 3(\bmod 4)$ if and only if $p \equiv 3(\bmod 8)$. Thus we have $\varepsilon_{\pi}=i$ and $-i$ respectively.

If $d \equiv 5(\bmod 8)$, we still have the above cases. There are four additional congruence classes for $\pi$ to consider, all of which have $l=0$. Two of these have $b^{\prime} \equiv 1$ and two have $b^{\prime} \equiv 3(\bmod 4)$.

If $d \equiv 2(\bmod 4)$, then, similarly, we consider $\left(\frac{2^{l+1}}{p}\right)$ if $b^{\prime} d^{\prime} \equiv 1(\bmod 4)$ and $-\left(\frac{2^{l+1}}{p}\right)$ if $b^{\prime} d^{\prime} \equiv 3(\bmod 4)$, where $d=2 d^{\prime}$. Since $p \equiv 3(\bmod 4)$, we have $l=0$. As above, in each case there is a correlation between $p(\bmod 8)$ and $b^{\prime} d^{\prime}(\bmod 4)$ that finishes this case.

Table 1. Values of $\varepsilon_{\pi}$ for $d \equiv 1(\bmod 4)$ and $\left(\frac{t}{\pi}\right)=\left(\frac{\sqrt{d}}{\pi}\right)$

\begin{tabular}{cccc}
\hline $\begin{array}{c}\text { Congruence class } \\
\text { of } \pi(\bmod 4)\end{array}$ & $d \equiv 1(\bmod 8)$ & $d \equiv 5(\bmod 16)$ & $d \equiv 13(\bmod 16)$ \\
\hline 1 & 1 & 1 & 1 \\
3 & 1 & 1 & 1 \\
$\frac{1+\sqrt{d}}{2}$ & $*$ & $i$ & 1 \\
$\frac{3+\sqrt{d}}{2}$ & $*$ & 1 & $i$ \\
$\frac{5+\sqrt{d}}{2}$ & $*$ & $i$ & 1 \\
$\frac{7+\sqrt{d}}{2}$ & $*$ & 1 & $i$ \\
$\sqrt{d}$ & $i$ & $i$ & $i$ \\
$2+\sqrt{d}$ & $-i$ & $-i$ & $-i$ \\
$\frac{1+3 \sqrt{d}}{2}$ & $*$ & $-i$ & 1 \\
$\frac{3+3 \sqrt{d}}{2}$ & $*$ & 1 & $i$ \\
$\frac{5+3 \sqrt{d}}{2}$ & $*$ & $-i$ & 1 \\
$\frac{7+3 \sqrt{d}}{2}$ & $*$ & 1 & $-i$ \\
\hline
\end{tabular}

* represents congruence classes that are not relatively prime to 4 . 
If $d \equiv 3(\bmod 4)$, then $\varepsilon_{\pi}=\left(\frac{2^{l}}{p}\right)$. Half of the classes have $l$ even and are thus 1 . When $l$ is odd, half of the classes have $p \equiv 1(\bmod 8)$ and half have $p \equiv 5(\bmod 8)$. Verifying this is mechanical.

The results of this appendix are best summarized in Tables 1 and 2. In the tables the value of $t$ is specified. A different choice of $t$ could change some of the Legendre symbol factors.

Table 2. Values of $\varepsilon_{\pi}$ for $d \equiv 2,3(\bmod 4)$

\begin{tabular}{|c|c|c|}
\hline $\begin{array}{l}\text { Congruence class } \\
\text { of } \pi(\bmod 4)\end{array}$ & $d \equiv 2(\bmod 4)$ & $d \equiv 3(\bmod 4)$ \\
\hline 1 & 1 & 1 \\
\hline 3 & 1 & 1 \\
\hline$\sqrt{d}$ & * & 1 \\
\hline $1+\sqrt{d}$ & $i$ & * \\
\hline $2+\sqrt{d}$ & * & 1 \\
\hline $3+\sqrt{d}$ & $i$ & $*$ \\
\hline $1+2 \sqrt{d}$ & 1 & -1 \\
\hline $3+2 \sqrt{d}$ & 1 & -1 \\
\hline $3 \sqrt{d}$ & $*$ & 1 \\
\hline $1+3 \sqrt{d}$ & $-i$ & $*$ \\
\hline $2+3 \sqrt{d}$ & $*$ & 1 \\
\hline $3+3 \sqrt{d}$ & $-i$ & $*$ \\
\hline
\end{tabular}

* represents congruence classes that are not relatively prime to 4 .

\section{References}

[1] S. Gelbart and I. I. Piatetski-Shapiro, Distinguished representations and modular forms of half-integral weight, Invent. Math. 59 (1980), 145-188.

[2] D. Gove, Modular forms of weight 1/2 over class number 1 imaginary quadratic number fields, Ph.D. Thesis, University of California, San Diego 1989.

[3] J.-P. Serre and H. M. Stark, Modular forms of weight 1/2, in: Lecture Notes in Math. 627, Springer, 1977, 27-67.

[4] G. Shimura, Introduction to the Arithmetic Theory of Automorphic Functions, Publ. Math. Soc. Japan 11, Princeton Univ. Press, 1971.

[5] H. M. Stark, Examples of modular forms over number fields, to appear.

DEPARTMENT OF MATHEMATICS

CALIFORNIA STATE UNIVERSITY

BAKERSFIELD, CALIFORNIA 93311

U.S.A.

E-mail:DGOVE@SPARC1.CSUBAK.EDU 